\title{
Slit-lamp Examination
}

National Cancer Institute

\section{Source}

National Cancer Institute. Slit-lamp Examination. NCI Thesaurus. Code C75583.

Examination of the anterior segment of the eye using a medical instrument called a slit lamp. 